\section{Crystal Structures of the Acid Salts of some Monocarboxylic Acids}

THE erystal structure of potassium hydrogen phenylacetate, $\mathrm{KH}\left(\mathrm{C}_{8} \mathrm{H}_{7} \mathrm{O}_{2}\right)_{2}$ (a substance already discussed in another connexion ${ }^{1}$ ), has been examined by X-ray analysis. The crystals belong to the monoclinic system, the unit cell having $a=28 \cdot 4 \mathrm{~A}$., $b=$ 4.50 A., $c=11 \cdot 9_{7}$ A., $\beta=89 \cdot 8^{\circ}\left( \pm 0 \cdot 3^{\circ}\right)$, and comprising four stoichiometric molecules. The spacegroup is $I 2 / a$ (that is, $C 2 / c$ of the International Tables with change of axes).

Using the isomorphous rubidium salt, a Patterson projection on the $(010)$ plane indicated provisional $x$ - and $y$-co-ordinates for the atoms; and, with this guidance, a corresponding Fourier synthesis has been carried out for the potassium compound. In the resulting projection, all eleven atoms of the asymmetric unit were well resolved, and the projected structure deduced is shown in the figure. Detailed consideration of this projection (and taking note of the strength of the reflexion from the (510) plane) gave a clue to the molecular orientation in the $y$-direction. The $\mathrm{C}-\mathrm{C}_{6} \mathrm{H}_{5}$ group lies in a plane inclined at about $31^{\circ}$ to $(010)$, and the $\mathrm{C}-\mathrm{CO}_{2}$ group in one inclined at about $36^{\circ}$ in the opposite direction. On this basis the complete structure has been elucidated, with some assistance from a Fourier projection on (001).

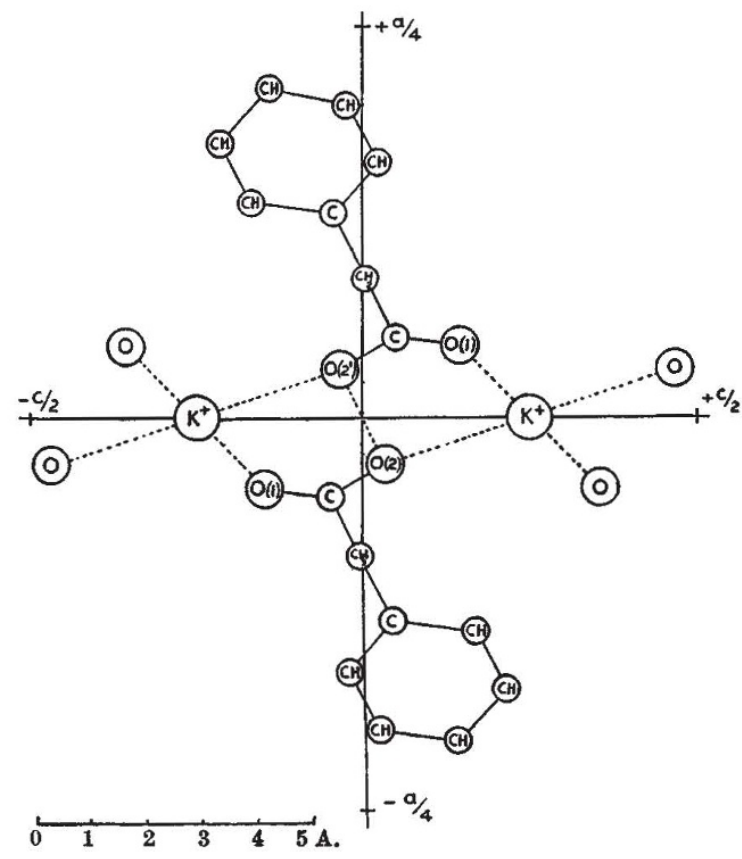

Interest is chiefly attached to the situation near the carboxyl groups. A hydrogen bond must be presumed to exist between the atoms $\mathrm{O}(2)$ and $\mathrm{O}\left(2^{\prime}\right)$; and, since these atoms lie about a centre of symmetry, the hydrogen atom appears to be centrally placed. All hydrogen bonds described hitherto seem to be unsymmetrical. It is possible that the virtual symmetry found here is a statistical effect. It is difficult to determine the $y$-co-ordinate of $\mathrm{O}(2)$ very accurately, but the true distance between the oxygen atoms is estimated to be $2 \cdot 5_{\mathrm{s}}( \pm 0 \cdot 1) \mathrm{A}$. This is unusually short (the dimers of carboxylic acids give values of about $2.7 \mathrm{~A}$.) and recalls the short bonds reported for same inorganic acid salts ${ }^{2}$ and for the dihydrates (probably oxonium salts) of some dibasic acids $^{3}$. Each potassium ion lies on a vertical twofold axis, and is surrounded by six approximately equidistant $(\sim 2.8$ A.) oxygen atoms which are situated at the corners of a distorted octahedron; two pairs, $\mathrm{O}(1)$, are superposed, at unit $b$-translation, in the projection shown. A number of different valency bond structures can be written, each lacking the necessary symmetry about the origin. The actual structure is taken to be a resonance hybrid, with consequent enhanced stability.

The structure of potassium hydrogen $p$-hydroxybenzoate, which is also being investigated, appears to be based on the same plan. Acid salts are formed by most monocarboxylic acids, and it seems likely that they may generally conform to this plan.

Further details of both structures will be published elsewhere.

\section{J. C. Speakman}

Chemistry Department,

University, Glasgow, W.2. Sept. 13.

${ }^{1}$ Speakman and Smith, Nature, 155, 698 (1945). A fuller report is in the press.

3 For example, West, Z. Krist., 74, 306 (1930). Zachariasen, J. Chem. Phys., 1, 634 (1933).

${ }^{3}$ For example, Dunitz and Robertson, J. Chem. Soc., 142, 148, 1145 (1947).

\section{Gamma-Rays from Proton Bombardment of Separated Argon Isotopes}

Argon targets can be prepared by bombarding silver disks with the separated beams of the argon isotopes in a mass-spectrograph. This technique has already been used in a study of the resonance capture of protons in the isotopes of neon ${ }^{1}$. The possibility of collecting argon atoms in a metal surface has previously been demonstrated ${ }^{2}$, and has recently been used as a method of producing samples of separated argon isotopes ${ }^{3}$.

With the abundant isotope ${ }^{40} \mathrm{~A}(99.632$ per cent) four targets were made which had received about 55 milli-coulombs per $\mathrm{cm}^{2}$ of ${ }^{40} \mathrm{~A}^{+}$-ions, and in addition one target which had received about 3 millicoulombs per em. ${ }^{2}$. For the measurements on ${ }^{36} \mathrm{~A}$ $(0 \cdot 307$ per cent) only one target, which had received about 3 milli-coulombs per cm. ${ }^{2}$, was made. No target was prepared containing the rare isotope ${ }^{38} \mathrm{~A}(0.061$ per cent), due to a contamination of the ion beam of mass 38 by fluorine molecular ions. The targets were used to search for capture resonances in the two argon isotopes for protons in the energy region from 0.5 to $1.8 \mathrm{MeV}$. The techniques of proton bombardment and gamma-ray detection were the same as those used in an earlier investigation*.

In the case of ${ }^{40} \mathrm{~A}$, six peaks were found standing out clearly above the background radiation, as determined with a dummy target. Five of these peaks are ascribed to ${ }^{40} \mathrm{~A}$. The accompanying table gives their resonance energies, and in addition the yields as measured with the four targets, which had been strongly bombarded with argon. Measurements of the resonance at $1,100 \mathrm{keV}$. with the weakly bombarded target gave half the yield, indicating that the targets are saturated with argon when they have received about 6 milli-coulombs per $\mathrm{cm}^{2}$, corresponding to about $2 \mu \mathrm{gm}$. per cm. ${ }^{2}$. The same saturation effect has also been found in the case of neon ${ }^{1}$. The half-widths of the peaks were in all cases $5-10 \mathrm{keV}$. 